BULL. AUSTRAL. MATH. SOC.

VOL. $19(1978), 183-190$.

\title{
Oscillatory behaviour of first order delay differential equations
}

\section{Alexander Tomaras}

Best possible conditions are given here, under which all solutions of several delay differential equations are oscillatory.

\section{Introduction}

The main interest in obtaining qualitative information for first order functional differential equations is due to the fact that they often provide mathematical models for physical systems (see, for instance, [1], $[3],[9],[12])$.

Of particular importance however has been the study of oscillations of delay differential equations, which are generated by the retarded argument and which do not appear in the corresponding differential equation without delay. Contributions in this area can be found, for example, in [2], [7], [8], while a special effort in the direction of establishing oscillation results of this kind for first order delay differential equations relevant to an industrial problem was undertaken in [10], [11].

Sufficient conditions, under which all solutions of the delay differential equation

$$
y^{\prime}(t)+p(t) y(t-\tau)=0
$$

are oscillatory, have been established by Ladas in [6], where it has also been explained that they are the best possible. Our aim here is to extend Ladas's results in [6] to the delay differential equation

Received 17 July 1978. 


$$
y^{\prime}(t)+p(t) y(g(t))=0 \text {. }
$$

Then the results obtained will be used to derive oscillatory information for the solutions of the delay differential equation:

$$
y^{\prime}(t)=p(t) y(g(t))+q(t) y(t),
$$

which is of more general form than an equation, arising as the mathematical idealization and simplification of an industrial problem, involving wave motion in the overhead supply line to an electrified railway system (see also [4], [5]). Both (1.2) and (1.3) have been considered in [10], but here, the results, which will be obtained for (1.2), and for (1.3) through (1.2), improve the results obtained in [10], and, in some cases, they are the best possible.

In the sequel a solution $y(t)$ of (1.2) or (1.3) is said to be oscizlatory, if it has arbitrarily large zeros, while it is said to be nonoscizlatory, if it is eventually of constant sign.

\section{Main results}

Assume that for (1.2), the conditions below are always true:

(CI) $p(t) \in C\left\{[0, \infty), R^{+}\right\}$;

(C2) $g(t) \in C^{1}\left\{[0, \infty), R^{+}\right\}, g(t)<t, \lim _{t \rightarrow \infty} g(t)=\infty$, and $g^{\prime}(t) \geq 0$

We state the following

THEOREM 2.1. In addition to ( $\mathrm{Cl}$ ) and (C2), suppose that the following conditions hold for (1.2):

(2.1) $\lim _{t \rightarrow \infty} \inf \int_{g(t)}^{t} p(s) d s>1 / e$;

(2.2) for every function $h(t) \in C^{1}\left\{[0, \infty), R^{+}\right\}$, such that

$$
\begin{aligned}
& g(t)<h(t)<t, \lim _{t \rightarrow \infty} h(t)=\infty, h^{\prime}(t) \geq 0, \text { the integral } \\
& \int_{h(t)}^{t} p(s) d s \text { is bounded away from zero. }
\end{aligned}
$$

Then every solution of (1.2) oscizlates. 
Proof. Let $y(t)$ be a nonoscillatory solution of (1.2), which we assume to be positive for $t>t_{0}, t_{0}$ being sufficiently large. Since $\lim _{t \rightarrow \infty} g(t)=\infty$, there exist,s a $t_{1}>t_{0}$, such that $y(g(t))>0$ and, from (1.2),

$$
y^{\prime}(t)<0, t>t_{1}
$$

Hence,

$$
y(t)<y(g(t)) \text { for } t>t_{2}>t_{1} \text {. }
$$

Set

$$
w(t)=y(g(t)) / y(t), \quad t>t_{2} .
$$

Then $w(t)>1$, and dividing both sides of (1.2) by $y(t)$ for $t>t_{2}$, we obtain

$$
y^{\prime}(t) / y(t)+p(t) w(t)=0, t>t_{2} .
$$

Integrating both sides of (2.4) from $g(t)$ to $t$, for $t>t_{3}>t_{2}$, we get

$$
\log y(t)-\log y(g(t))+\int_{g(t)}^{t} p(s) w(s) d s=0, \quad t>t_{3},
$$

or, in view of $(2.3)$,

$$
\log w(t)=\int_{g(t)}^{t} p(s) w(s) d s, t>t_{3} .
$$

Set

$$
\lim _{t \rightarrow \infty} \inf w(t)=m
$$

Then $m \geq !$, and it is finite or infinite.

CASE $1 . m$ is finite. Then there exists a sequence $t_{n} \rightarrow \infty$, such that $\lim _{n \rightarrow \infty}$ inf $w\left(t_{n}\right)=m$. From (2.5), we get 
(2.7) $\log w\left(t_{n}\right)=\int_{g\left(t_{n}\right)}^{t_{n}} p(s) w(s) d s=w\left(v_{n}\right) \int_{g\left(t_{n}\right)}^{t_{n}} p(s) d s$, where $g\left(t_{n}\right)<v_{n}<t_{n}, n=1,2, \ldots$.

Taking limits on both sides of (2.7), as $n \rightarrow \infty$, and account of (2.6), we obtain

$$
\log m \geq m \lim _{t \rightarrow \infty} \inf \int_{g(t)}^{t} p(s) d s,
$$

and so

$$
(\log m) / m \geq \lim _{t \rightarrow \infty} \inf \int_{g(t)}^{t} p(s) d s .
$$

Using the fact that

$$
\max _{m \geq 1}(\log m) / m=1 / e
$$

(2.8) implies

$$
1 / e \geq \lim _{t \rightarrow \infty} \inf \int_{g(t)}^{t} p(s) d s,
$$

which contradicts (2.1).

CASE 2. $m=+\infty$. That is,

$$
\lim _{t \rightarrow \infty} y(g(t)) / y(t)=+\infty \text {. }
$$

Let then $h(t)$ be a function as in (2.2).

Integrating both sides of (1.1) from $h(t)$ to $t>t_{4}>t_{3}$, for sufficiently large $t_{4}$, we obtain

$$
y(t)-y(h(t))+\int_{h(t)}^{t} p(s) y(g(s)) d s=0 .
$$

Since $y(g(s))>y(g(t))$ for $h(t) \leq s \leq t,(2.10)$ yields

$$
y(t)-y(h(t))+y(g(t)) \int_{h(t)}^{t} p(s) d s \leq 0 .
$$


Dividing both sides of (2.11) by $y(t)$, taking limits, as $t \rightarrow \infty$, and using (2.9) and (2.2), we conclude that

$$
\lim _{t \rightarrow \infty} y(h(t)) / y(t)=+\infty \text {. }
$$

Dividing both sides of (2.11) by $y(h(t))$, we get

$$
y(t) / y(h(t))-1+y(g(t)) / y(h(t)) \int_{h(t)}^{t} p(s) d s \leq 0,
$$

which, if we take limits, as $t \rightarrow \infty$, in view of (2.12) and (2.2), is impossible. Since in both cases we are led to a contradiction, the proof of the theorem is complete.

Let us now look at (1.3). In [10] it is shown that (1.3) can be led to the form

$$
z^{\prime}(t)+Z(t) z(g(t))=0
$$

if we set

$$
y(t) \exp \left[-\int q(t) d t\right]=z(t)
$$

and

$$
Z(t)=-p(t) \exp \int_{t}^{g(t)} q(T) d T
$$

and that the oscillatory character of its solutions is maintained under the above transformations.

Since (2.13) is the same as (1.2), we apply the previous theorem to it, to establish the following result for (1.3).

THEOREH 2.2. Consider the delay differential equation (1.3), subject to the following conditions:

$$
\begin{aligned}
& \text { (i) }-p(t) \in C\left\{[0, \infty), R^{+}\right\} ; \\
& \text {(ii) } g(t) \in C^{1}\left\{[0, \infty), R^{+}\right\}, g(t)<t, \lim _{t \rightarrow \infty} g(t)=\infty \text {, and } \\
& g^{\prime}(t) \geq 0 ; \\
& \text { (iii) } q(t) \text { is continuous for any } t \neq 0 . \\
& \text { If, in addition, }
\end{aligned}
$$




$$
\lim _{t \rightarrow \infty} \inf \int_{g(t)}^{t}-p(s)\left[\exp \int_{s}^{g(s)} q(T) d T\right] d s>1 / e
$$

and

(2.15) for every function $h(t) \in C^{1}\left\{[0, \infty), R^{+}\right\}$, such that $g(t)<h(t)<t, \lim _{t \rightarrow \infty} h(t)=\infty, h^{\prime}(t) \geq 0$, the integral $\int_{h(t)}^{t}-p(s)\left[\exp \int_{s}^{g(s)} q(T) d T\right] d s$ is bounded away from zero,

then every solution $y(t)$ of (1.3) oscillates.

DISCUSSION. Conditions (2.1), (2.14), and (2.2), (2.15) are sufficient in Case 1 and Case 2 respectively, for all solutions of (1.2) and (1.3) to oscillate.

If $p(t)$ is a positive constant $p$, that is, in the case of the delay differential equation

$$
y^{\prime}(t)+p y(g(t))=0,
$$

condition (2.1) becomes

$$
\lim _{t \rightarrow \infty} \inf [t-g(t)]>1 / p e
$$

and, if in addition, $g(t)=t-\tau$, where $\tau$ is a positive constant, it becomes

$$
\text { pre }>1 \text {. }
$$

Then, clearly, (2.18) is the best possible condition for all solutions of the delay differential equation

$$
y^{\prime}(t)+p y(t-\tau)=0
$$

to oscillate (this has been established by Ladas in [6]), and (2.17) is the best possible condition for all solutions of the delay differential equation (2.16) to oscillate, in Case 1. Accordingly, in the same case, (2.1) is the best possible condition for all solutions of (1.2) to oscillate, and $(2.14)$ is the best possible condition for all solutions of (1.3) to oscillate.

It is to be noted that in Case $1,(2.14)$ is weaker than condition 
(2.4) in [10]. On the other hand, Theorem 2.1 here, for (1.2), shows to be not just an adaptation of Ladas's results in [6], for (1.1). This means that results for nonconstant delays cannot be expected to occur immediately from results concerning constant delays. Also, (1.2) has been again the key to oscillatory information for (1.3) (see the relevant technique in [10]).

Finally, the question "how far away from zero" the integrals in (2.2) and (2.15) should be bounded, remains open.

\section{References}

[1] Kenneth L. Cooke and James A. Yorke, "Equations modelling population growth, economic growth, and gonorrhea epidemiology", Ordinary differential equations, 1971 NRL-MRC Conference, 35-53 (Proc. Conf. Ordinary Differential Equations held Washington, D.C., 1971. Academic Press, New York and London, 1972).

[2] R.D. Driver, "Linear differential systems with small delays", $J$. Differential Equations 21 (1976), 148-166.

[3] L. Fox, D.F. Mayers, J.R. Ockendon and A.B. Tayler, "On a functional differential equation", J. Inst. Math. Appl. 8 (1971), 271-307.

[4] Tosio Kato, "Asymptotic behaviour of solutions of the functional differential equation $y^{\prime}(x)=a y(\lambda x)+b y(x)$ ", Delay and functional differential equations and their applications, 197-217 (Proc. Conf. Park City, Utah, 1972. Academic Press, New York, London, 1972).

[5] Tosio Kato and J.B. McLeod, "The functional-differential equation $y^{\prime}(x)=a y(\lambda x)+b y(x)$ ", Bulz. Amer. Math. Soc. 77 (1971), $891-937$.

[6] Gerasimos Ladas, "Sharp conditions for oscillations caused by delays" (Technical Report, 64. Department of Mathematics, University of Rhode Island, Kingston, Rhode Island, 1976). 
[7] G. Ladas, V. Lakshmikantham, and J.S. Papadakis, "Oscillations of higher-order retarded differential equations generated by the retarded argument", Delay and functional differential equations and their applications, 219-231 (Proc. Conf. Park City, Utah, 1972. Academic Press, New York, London, 1972).

[8] James C. Lillo, "Oscillatory solutions of the equation $y^{\prime}(x)=m(x) y(x-n(x))$ ", J. Differential Equations 6 (1969), $1-35$.

[9] J.R. Ockendon and A.B. Tayler, "The dynamics of a current collection system for an electric locomotive", Proc. Roy. Soc. London A 322 $(1971), 447-468$.

[10] Alexander Tomaras, "Oscillations of an equation relevant to an industrial problem", Bull. Austral. Matn. Soc. 12 (1975), 425-431.

[11] Alexander Tomaras, "Oscillatory behaviour of an equation arising from an industrial problem", Bulz. Austral. Hath. Soc. 13 (1975), $255-260$.

[12] James A. Yorke, "Selected topics in differential delay equations", Japan-United States Seminar on Ordinary Differential and Functional Equations, 16-28 (Lecture Notes in Mathematics, 243. Springer-Verlag, Berlin, Heidelberg, New York, 1971).

\footnotetext{
Mathematical Institute,

University of Oxford,

Oxford,

England.
} 